\title{
Enhanced exosomes and spinal cord ischemia: Excitement and a Nobel Prize but more to come
}

\author{
Victor A. Ferraris, MD, PhD
}

From the Department of Surgery, University of Kentucky, Lexington, Ky.

Disclosures: Author has nothing to disclose with regard to commercial support.

Received for publication June 27, 2018; accepted for publication June 28, 2018; available ahead of print Aug 14, 2018.

Address for reprints: Victor A. Ferraris, MD, PhD, Department of Surgery, University of Kentucky, A301 Kentucky Clinic, 740 S Limestone, Lexington, KY 40536-0284 (E-mail: ferraris@uky.edu).

J Thorac Cardiovasc Surg 2019;157:518-9

0022-5223/\$36.00

Copyright (c) 2018 by The American Association for Thoracic Surgery

https://doi.org/10.1016/j.jtcvs.2018.06.085

In 2013, Americans James Rothman and Randy Schekman and German-born researcher Thomas Sudhof won the Nobel Prize in Medicine for discoveries on how hormones, enzymes, and other key substances are transported within and between cells. In the decades between the 1970s and 1990s, Schekman discovered a set of genes that were required for vesicle transport, Rothman found how vesicles delivered their cargo to the right places, and Sudhof identified the machinery that controls when vesicles release chemical messengers from a brain cell that allow it to communicate with another.

Exosomes are a subtype of these transport vesicles that are released by almost all cell types and are present in most, if not all, biologic fluids. The biologically active cargo carried by exosomes, especially micro-RNA (miRNA) can alter the phenotype of recipient cells. Exosomes have an important role in the progression and treatment of cardiac disease states, neurologic dysfunction, oncogenesis, and metabolic disorders. Injured cardiac cells can release exosomes with important pathologic effects on surrounding tissue. Perhaps more importantly, it is possible that exosomes released from stem cells can facilitate repair of nerve cell damage in certain organ systems, including the central nervous system.

Exosomes contain materials that might convey messages between cells. In their work leading up to the Nobel Prize, Schekman and colleagues ${ }^{2}$ developed a method to better isolate exosomes from cells to examine their contents. They found that some RNA molecules are hundreds or thousands of times more concentrated in exosomes than in the cells they come from. For example, cancer cells could secrete exosomes to promote cell and blood vessel growth that facilitate tumor progression. Schekman suspects that cells have a way to sort specific RNA molecules into exosomes, and his lab found factors, including specific proteins, that cells require to package these molecules.

Exosomes contain miRNAs, a class of naturally occurring small noncoding RNA sequences that can regulate a recipient cell's signaling pathways and alter protein

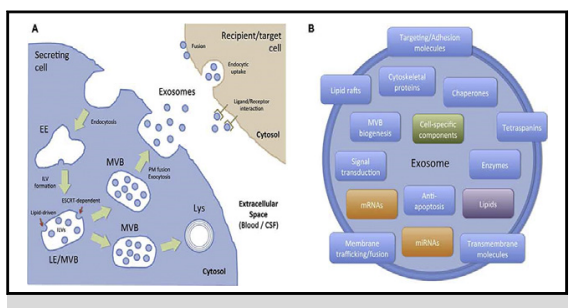

Mechanisms for exosome creation.

Central Message

Exosomes contain materials that might convey messages between cells. Their potential is enormous but unintended consequences exist.

See Article page 508

expression, either acting alone or together with other miRNAs. ${ }^{3}$ In addition, miRNAs are scavengers. Evidence suggests that miRNAs play a role in the purging and transferring excess free RNAs, including fragmented transfer RNAs and other small noncoding RNAs. ${ }^{2}$

These exciting and abundant experimental results, some of which were recognized by the Nobel Committee, triggered speculations about the potential clinical benefit of exosome-related mediation of cell function in key metabolic, and perhaps dysfunctional, pathways. This excitement has mostly escaped application in human trials. Zhao and colleagues ${ }^{4}$ present their work on modification of exosomes derived from bone marrow mesenchymal stem cells that over express miRNA-25. The authors chose miRNA-25 because of its negative regulatory effect on NOX4-mediated oxidative stress in neurologic tissue. The authors' efforts to overexpress miRNA-25 in mesenchymal stem cells is novel and groundbreaking. In their article, ${ }^{4}$ they delivered increased amounts of miRNA-25 enhanced exosomes to spinal cords in a rat model of ischemic spinal cord stress. They found that these enhanced exosomes provided neuroprotection in their model.

The cargoes carried by exosomes are complex and their only unifying characteristic is that the cargoes must fit into a confined space of no more than $100 \mathrm{~nm}$. The size of miRNAs is usually about the length of 20 nucleic acids, ideal for cargo within exosomes. This size limitation is both an advantage and disadvantage. Larger-size molecules, including some transfer RNAs (typically 70-90 nucleotides), and other macromolecules are mostly excluded 
from the content of miRNAs. Ironically, most exosomes derived from standard preparations do not harbor many copies of miRNA molecules. ${ }^{5}$ This small size of miRNAs provides some advantages. ${ }^{6}$ For example, these small exosomes are ideal candidates for noninvasive indicators of pathogenesis or even indicators of disease progression. The miRNA content of exosomes, including individualized nucleic acid, protein, and lipid expression profiles, can indicate a disease state or even serve as tumor markers. Furthermore, the ability of exosomes to carry signaling molecules to distant tissues suggests these particles may provide an avenue for delivery of therapeutic treatments to target areas, particularly in cancer, neurodegenerative diseases, or ischemic stroke. ${ }^{7,8}$ Exosomes that contain Alzheimer disease-specific oligomeric proteins have potential to provide ultrasensitive early detection of Alzheimer disease. ${ }^{7,9}$

However, the exciting possibilities attributed to enhanced exosomes must be tempered by some harsh realities. Disadvantages associated with exosomes abound in the literature. First, analytic methods for harvest and analysis of exosomes are not simple. ${ }^{10,11}$ Perhaps the most important concern revolves around unintended consequences of enhanced miRNAs carried by exosomes. Exosomes can generate a protumor environment that is essential for tumorigenesis. ${ }^{12-16}$ It is uncertain that modification of exosomes to overexpress 1 miRNA will only have a very specific influence and may in fact promote undesirable metabolic pathways with adverse outcomes.

The authors used appropriate caution in the presentation of their results on the effects of enhanced exosomes on spinal cord ischemia. This caution should not detract from the potential benefits that seem to be just around the corner of this interesting, exotic, and Nobel Prize-awarded avenue for research and potential human trials. I will be surprised if some dramatic advancements in any of several areas, including drug delivery, modification of tumorogenesis, limitations of ischemic injury, and enhancement of other basic physiologic processes, do not surface from the further study of miRNA-enhanced exosomes. I look forward to future work in this area.

\section{References}

1. Poe AJ, Knowlton AA. Exosomes and cardiovascular cell-cell communication. Essays Biochem. 2018;62:193-204.

2. Shurtleff MJ, Yao J, Qin Y, Nottingham RM, Temoche Diaz MM, Schekman R, et al. Broad role for YBX1 in defining the small noncoding RNA composition of exosomes. Proc Natl Acad Sci U S A. 2017;114:E8987-95.

3. Venkat P, Chopp M, Chen J. Cell-based and exosome therapy in diabetic stroke. Stem Cells Transl Med. 2018;7:451-5.

4. Zhao L, Jiang X, Shi J, Gao S, Zhu Y, Gu T, Shi E. Exosomes derived from bone marrow mesenchymal stem cells overexpressing microRNA-25 protect spinal cords against transient ischemia. J Thorac Cardiovasc Surg. 2019;157:508-17.

5. Chevillet JR, Kang Q, Ruf IK, Briggs HA, Vojtech LN, Hughes SM, et al. Quantitative and stoichiometric analysis of the microRNA content of exosomes. Proc Natl Acad Sci U S A. 2014;111:14888-93.

6. Tickner JA, Richard DJ, O'Byrne KJEV. Microvesicles/microRNAs and stem cells in cancer. Adv Exp Med Biol. 2018;1056:123-35.

7. Venugopal C, Shamir C, Senthilkumar S, Babu JV, Sonu PK, Nishtha K, et al. Dosage and passage dependent neuroprotective effects of exosomes derived from rat bone marrow mesenchymal stem cells: an in vitro analysis. Curr Gene Ther. 2017;17:379-90.

8. Manuel GE, Johnson T, Liu D. Therapeutic angiogenesis of exosomes for ischemic stroke. Int J Physiol Pathophysiol Pharmacol. 2017;9:188-91.

9. Zhou J, Meng L, Ye W, Wang Q, Geng S, Sun C. A sensitive detection assay based on signal amplification technology for Alzheimer's disease's early biomarker in exosome. Anal Chim Acta. 2018;1022:124-30.

10. Tang YT, Huang YY, Zheng L, Qin SH, Xu XP, An TX, et al. Comparison of isolation methods of exosomes and exosomal RNA from cell culture medium and serum. Int J Mol Med. 2017;40:834-44.

11. Ding M, Wang C, Lu X, Zhang C, Zhou Z, Chen X, et al. Comparison of commercial exosome isolation kits for circulating exosomal microRNA profiling. Anal Bioanal Chem. 2018;410:3805-14.

12. Wang JP, Tang YY, Fan CM, Guo C, Zhou YH, Li XL, et al. The role of exosomal non-coding RNAs in cancer metastasis. Oncotarget. 2018;9:12487-502.

13. Wang X, Luo G, Zhang K, Cao J, Huang C, Jiang T, et al. Hypoxic tumor-derived exosomal miR-301a mediates M2 macrophage polarization via PTEN/PI3Kgamma to promote pancreatic cancer metastasis. Cancer Res. 2018;78:4586-98.

14. Lin XJ, Fang JH, Yang XJ, Zhang C, Yuan Y, Zheng L, et al. Hepatocellular carcinoma cell-secreted exosomal microRNA-210 promotes angiogenesis in vitro and in vivo. Mol Ther Nucleic Acids. 2018;11:243-52.

15. Kwizera EA, O'Connor R, Vinduska V, Williams M, Butch ER, Snyder SE, et al Molecular detection and analysis of exosomes using surface-enhanced raman scattering gold nanorods and a miniaturized device. Theranostics. 2018;8: 2722-38.

16. Al Saleh HA, Haas-Neill S, Al-Hashimi A, Kapoor A, Shayegan B, Austin R, et al. Thrombotic characteristics of extracellular vesicles derived from prostate cancer cells. Prostate. 2018;78:953-61. 